Nouvelles perspectives en sciences sociales

Revue internationale de systémique complexe et d'études relationnelles

\title{
Prolégomènes à une linguistique de la complexité
} Avant-propos

\section{Ali Reguigui et Julie Boissonneault}

Volume 9, numéro 1, novembre 2013

Sur le thème : linguistique et complexité

URI : https://id.erudit.org/iderudit/1024037ar

DOI : https://doi.org/10.7202/1024037ar

Aller au sommaire du numéro

Éditeur(s)

Prise de parole

ISSN

1712-8307 (imprimé)

1918-7475 (numérique)

Découvrir la revue

Citer ce document

Reguigui, A. \& Boissonneault, J. (2013). Prolégomènes à une linguistique de la complexité : avant-propos. Nouvelles perspectives en sciences sociales, 9(1),

13-16. https://doi.org/10.7202/1024037ar d'utilisation que vous pouvez consulter en ligne. 


\title{
Prolégomènes à une linguistique de la complexité
}

\section{Avant-propos}

\author{
Ali Reguigui \\ Université Laurentienne, Sudbury \\ JuLIE BoIsSONNEAULT \\ Université Laurentienne, Sudbury
}

près avoir marqué le $\mathrm{XX}^{\mathrm{e}}$ siècle, la linguistique se trouve à
la croisée des chemins. Le succès qu'elle a connu est tel que son objet d'étude est devenu multiplexe. Or, cet objet ne peut plus être occulté et doit désormais être considéré dans l'apparent désordre et dans la complexité qui le génèrent. La linguistique est ainsi aujourd'hui en quête d'une nouvelle identité et d'un nouvel objet. Comment peut-elle négocier les besoins de l'objectivation et de l'ordre, d'une part, et les impératifs du désordre et de la complexité, d'autre part? Ce sont là des questions auxquelles certains membres de la communauté des chercheurs ont tenté de répondre en posant une réflexion, ayant pour toile de fond aussi bien la théorie que l'empirie, de nature à mettre les premiers jalons pour une linguistique du XXI siècle. Ce numéro de Nouvelles perspectives en sciences sociales se veut donc une contribution à l'étude de la complexité plutôt qu'une réponse exhaustive à toutes les questions. 
Le champ de la linguistique ne désemplie pas de tentatives qui visent à dénouer l'impasse créée par la complexification des approches nées de la surspécialisation et de l'hyperspécialisation qui, tout en raffinant la connaissance des objets d'étude, ont permis de découvrir les limites de celles-ci. Parmi ces approches, notons la recherche de méthodes capables de rendre compte de la complexité quelle que soit la langue. Les développements considérables dans les domaines de la linguistique cognitive, de la linguistique de corpus et de la linguistique computationnelle ont permis, chacune d'une perspective différente, d'apporter une contribution à l'analyse de cette complexité. L'essai d'Amr Helmy Ibrahim s'inscrit dans cette démarche. Il soutient la possibilité de fonder une méthode descriptive métalinguistique qui utilise des paramètres normalisés susceptibles de mesurer la complexité linguistique et qui vise la décomplexification des phénomènes complexes.

Dans un essai qui tente de négocier un équilibre entre les besoins de la spécialisation et ceux de la vulgarisation, Sara Vecchiato et Sonia Vanna Gerolimich dressent une échelle d'analyse d'une langue de spécialité - le langage médical -, oscillant entre deux pôles de la complexité. Cet équilibre est difficile à atteindre surtout quand on se trouve confronté, au-delà de la spécialisation, à une surspécialisation poussée à une hyperspécialisation, paralysant ainsi la communication entre experts eux-mêmes. Que dire alors du mur d'opacité informationnelle complète dressé face au profane.

La complexité est non seulement un phénomène macrolinguistique, mais elle se trouve jusque dans les infimes parties de la langue et de tout ce qui l'implique. Ainsi, le phénomène d'acquisition langagière a connu des développements sans cesse grandissants depuis l'émergence de la psycholinguistique et, grâce à la contribution de diverses disciplines connexes, nous sommes à même d'observer des effets d'interaction qu'il aurait été impossible d'imaginer il y a à peine deux décennies. Dans la foulée de ces développements, Claire Martinot explore la complexité du développement du langage en se fondant sur 
l'étude comparative de l'acquisition des relatives en langue première auprès d'enfants français et allemands. Son étude permet de découvrir des critères indirects mais déterminants sur l'acquisition des relatives.

Mais si la complexité formelle aura toujours pour corolaire les formes et leurs interactions tant intralinguistiques qu'extralinguistiques, le sens demeure un élément évasif dont l'interprétation est tributaire de la diachronie, de l'usage, du contexte, de la situation et même des variations idiolectales. À partir de l'analyse d'opérateurs d'intensité, Clara Romero fonde une définition générale de la complexité sémantique et conclut à une hiérarchisation de celle-ci. Pour sa part, Brahime Larouz centre son attention sur le problème de la référentialité en indiquant que l'énoncé partage cette fonction avec le contexte d'énonciation et que les phrases sont porteuses d'indices sur les aspects situationnels à considérer pour nous aiguiller vers le référent.

La complexité linguistique s'accroît quand les divers niveaux d'analyse et les diverses disciplines se chevauchent et que la synchronie fait écho à la diachronie tout en rompant avec elle par l'effet de la variation. Dans cette perspective, Nizha ChatarMoumni explore les fondements historiques de la négation en arabe marocain afin d'en comprendre les usages synchroniques. Par ailleurs, Danh Thành Do-Hurinville, qui soutient que les langues diffèrent par les lieux et les instances de complexité, présente cette prise de position par l'analyse distributionnelle des diverses unités du vietnamien aux niveaux morpho-phonologique, syntactico-sémantique et discursif. Ce faisant, elle montre comment la polycatégorialité des unités d'analyse constitue un caractère particulier de la complexité linguistique du vietnamien.

Enfin, Louis-Jean Calvet présente d'autres dimensions de la complexité linguistique en se penchant sur la problématique du signe chinois. En effet, à partir de questionnements posés par Lacan sur le signe chinois, la psychanalyse et le rêve, il explore le concept de signe chinois à la lumière du signe saussurien et du principe de linéarité. Compte tenu de la capacité d'évocation polyphonique du même signe et de la présence de segments 
graphiques non reliés aux sens exprimés, mais plutôt reliés à l'histoire, à la symbolique et à l'imaginaire chinois, l'analyse prend un virage interdisciplinaire alliant, entre autres, la synchronie, la diachronie, la psychanalyse, la sociologie, l'anthropologie.

Ce numéro sur la linguistique comme science de la complexité ne fait qu'effleurer la question de la complexité à l'œuvre. Nous sommes loin d'avoir développé une méthode d'analyse capable de rendre compte de toutes les complexités. Nous sommes également loin de connaître l'ampleur et la diversité de cette complexité. Trouvons consolation dans le fait que la recherche continuera à aller de l'avant et que l'avenir est prometteur de beaux défis et de belles découvertes. 